\title{
The strong mixing and the selfdecomposability properties
}

\author{
Richard C. Bradley* and Zbigniew J. Jurek ${ }^{\dagger} \ddagger$
}

July 21, 2013

\begin{abstract}
It is proved that infinitesimal triangular arrays obtained from normalized partial sums of strongly mixing (but not necessarily stationary) random sequences, can produce as limits only selfdecomposable distributions.
\end{abstract}

Mathematics Subject Classifications(2010): Primary 60B10, 60B12, 60E07.

Key words and phrases: Strongly mixing sequence; infinitesimal triangular array; selfdecomposable distribution; Banach space.

Abbreviated title: Strong mixing and selfdecomposability

Selfdecomposable probability measures (in other words, the Lévy class L distributions) form (by definition) the class of possible limiting distributions of normalized partial sums from sequences of independent (but not necessarily identically distributed) random variables, under certain natural technical assumptions on the normalizing constants. The aim of this note is to show that selfdecomposable probability measures also form the class of possible limiting distributions of normalized partial sums from (not necessary stationary) strongly mixing sequences, under the same technical assumptions on the normalizing constants. The proof will utilize the standard Bernstein blocking technique.

${ }^{*}$ Department of Mathematics, Indiana University, Bloomington, Indiana 47405, USA.

†Institute of Mathematics, University of Wrocław, Pl. Grunwaldzki 2/4, 50-384 Wrocław, Poland.

${ }^{\ddagger}$ Research funded by Narodowe Centrum Nauki (NCN) grant no Dec2011/01/B/ST1/01257. 
For normalized partial sums from strictly stationary, strongly mixing random sequences, with a mild natural assumption on the normalizing constants, two other classes of distributions - the stable and infinitely divisible laws have long been known to play the same roles respectively as they do in the case of i.i.d. sequences: as possible limit laws (i) along the entire sequence of normalized partial sums, and (ii) along subsequences of normalized partial sums. For further information and references on those classic results, see e.g. Volume 1, Chapter 12 of Bradley (2007). We shall not treat the particular case of strict stationarity further here.

\section{Notations and basic notions.}

Let $(\Omega, \mathcal{F}, P)$ be a probability space. Let $E$ be a real separable Banach space, with norm $\|\cdot\|$ and Borel sigma-algebra $\mathcal{E}$. By $\mathcal{P} \equiv \mathcal{P}(E)$ we denote the set of all Borel probability measures on $E$, with the convolution operation denoted by "*" and weak convergence denoted by " $\Rightarrow$ ", which make $\mathcal{P}$ a topological convolution semigroup.

Measurable functions $\xi: \Omega \rightarrow E$ are called Banach space valued random variables (in short: $E$-valued rv's) and $\mathcal{L}(\xi)(A):=P\{\omega \in \Omega: \xi(\omega) \in A\}$, for $A \in \mathcal{E}$, is the probability distribution of $\xi$. Then for stochastically independent $E$-valued random variables $\xi_{1}$ and $\xi_{2}$ we have that $\mathcal{L}\left(\xi_{1}\right) * \mathcal{L}\left(\xi_{2}\right)=\mathcal{L}\left(\xi_{1}+\right.$ $\left.\xi_{2}\right)$. Also for $c \in \mathbb{R} \backslash\{0\}$ and rv $\xi$ we define $\mathcal{L}(c \xi)(A)=\mathcal{L}(\xi)\left(c^{-1} A\right)=$ : $T_{c}(\mathcal{L}(\xi))(A)$, for $A \in \mathcal{E}$. Similarly, for $T_{c}: E \rightarrow E$ given by $T_{c} x:=c x$, we define $T_{c} \mu$, for $\mu \in \mathcal{P}$. Hence $T_{c}(\mu * \nu)=T_{c} \mu * T_{c} \nu$.

For two sub- $\sigma$-fields $\mathcal{A}$ and $\mathcal{B}$ of $\mathcal{F}$ we define the measure of dependence $\alpha$ between them as follows:

$$
\alpha(\mathcal{A}, \mathcal{B}):=\sup _{A \in \mathcal{A}, B \in \mathcal{B}}|P(A \cap B)-P(A) P(B)| .
$$

For a given sequence $\mathbf{X}:=\left(X_{1}, X_{2}, \ldots\right)$ of $E$-valued random variables, we define for each positive integer $n$ the dependence coefficient

$$
\alpha(n) \equiv \alpha(\mathbf{X} ; n):=\sup _{j \in \mathbf{N}} \alpha\left(\sigma\left(X_{k}, 1 \leq k \leq j\right), \sigma\left(X_{k}, k \geq j+n\right)\right),
$$

where $\sigma(\ldots)$ denotes the $\sigma$-field generated by $(\ldots)$. We will say that a sequence $\mathbf{X}$ is strongly mixing (Rosenblatt (1956)) if

$$
\alpha(n) \rightarrow 0 \text { as } n \rightarrow \infty
$$

Of course, if the elements of $\mathbf{X}$ are stochastically independent then $\alpha(\mathbf{X} ; n) \equiv$ 0 . Many known stochastic processes (including many Markov chains, many Gaussian sequences, and many models from time series analysis) have long been known to be strongly mixing; see e.g. Bradley (2007). 
Suppose that for stochastically independent $E$-valued rv's $\xi_{j}, j \in \mathbf{N}$, there exist sequences of real numbers $a_{n}$ and vectors $b_{n} \in E$ and a probability measure $\nu$ such that

$$
\text { (i) } a_{n}>0 \text { and } \forall(\epsilon>0) \lim _{n \rightarrow \infty} \max _{1 \leq k \leq n} P\left(\left\{\omega \in \Omega: a_{n}\left\|\xi_{k}(\omega)\right\|>\epsilon\right\}\right)=0
$$

(the so called infinitesimality condition) and

$$
\text { (ii) } \lim _{n \rightarrow \infty} P\left(\left\{\omega \in \Omega: a_{n}\left(\xi_{1}+\xi_{2}+\cdots+\xi_{n}\right)(\omega)+b_{n} \in B\right\}\right)=\nu(B)
$$

for every Borel set $B \subset E$ whose boundary $\partial B$ satisfies $\nu(\partial B)=0$; then the measure $\nu$ is called selfdecomposable or a Lévy class $L$ distribution.

There are two basic characterizations of the class $L$ : the convolution decomposition and the random integral representation. The first one says that

$$
[\nu \in L] \text { iff }\left[\forall(0<c<1) \exists\left(\nu_{c} \in \mathcal{P}(E)\right) \quad \nu=T_{c} \nu * \nu_{c}\right] \text {, }
$$

and hence the term selfdecomposability; cf. Jurek and Mason (1993), Theorem 3.9.2.

The second one says that

$$
[\nu \in L] \text { iff } \nu=\mathcal{L}\left(\int_{0}^{\infty} e^{-t} d Y_{\rho}(t)\right)
$$

for some Lévy process $\left(Y_{\rho}(t), t \geq 0\right)$ such that $\mathcal{L}\left(Y_{\rho}(1)\right)=\rho$ and the logmoment $\mathbb{E}\left[\log \left(1+\left\|Y_{\rho}(1)\right\|\right)\right]<\infty$; cf. Jurek and Vervaat (1983) or Jurek and Mason (1993), Theorem 3.9.3. The Lévy process $Y_{\rho}$ in (6) is referred to as the background driving Lévy process (in short: BDLP) of the selfdecomposable probability measures $\nu$.

Finally let us note that in terms of probability measures, (4) means that

$$
T_{a_{n}}\left(\rho_{1} * \rho_{2} * \ldots * \rho_{n}\right) * \delta_{b_{n}} \Rightarrow \nu \text { as } n \rightarrow \infty
$$

where $\rho_{i}=\mathcal{L}\left(\xi_{i}\right)$ for $i=1,2, \ldots$ For probability theory on Banach spaces we refer to Araujo and Giné (1980).

\section{Strong mixing and selfdecomposability.}

Here is the main result of this note:

THEOREM 1. Let $\boldsymbol{X}:=\left(X_{1}, X_{2}, \ldots\right)$ be a sequence of Banach space $E$ valued random variables with partial sums $S_{n}:=X_{1}+X_{2}+\cdots+X_{n}$, and let $\left(a_{n}\right)$ and $\left(b_{n}\right)$ be sequences of real numbers and elements in $E$ respectively, and suppose the following conditions are satisfied: 
(i) $\alpha(n) \rightarrow 0$ as $n \rightarrow \infty$, i.e. the sequence $\boldsymbol{X}$ is strongly mixing;

(ii) $a_{n}>0$ and the triangular array $\left(a_{n} X_{j}, 1 \leq j \leq n, n \geq 1\right)$ is infinitesimal;

(iii) $a_{n} S_{n}+b_{n} \Rightarrow \mu$ as $n \rightarrow \infty$ for some non-degenerate probability measure $\mu \in \mathcal{P}$.

Then the limit distribution $\mu$ is selfdecomposable.

Proof of theorem. Our aim is to show that $\mu$ satisfies the convolution decomposition (5). The argument below is divided into a few steps/observations, some of which are quite elementary.

Step 1. $a_{n} \rightarrow 0$ and $a_{n+1} / a_{n} \rightarrow 1$ as $n \rightarrow \infty$.

Proof. a) Let us first show that $a_{n} \rightarrow 0$. Suppose instead that $Q$ is an infinite subset of $\mathbf{N}, d>0$, and $a_{n}>d$ for all $n \in Q$. Then for all $\epsilon>0$

$$
\max _{1 \leq k \leq n} P\left(d\left|X_{k}\right|>\epsilon\right) \leq \max _{1 \leq k \leq n} P\left(a_{n}\left|X_{k}\right|>\epsilon\right) \rightarrow 0 \text { as } n \rightarrow \infty, n \in Q .
$$

Thus for each fixed $k$ and each $\epsilon>0$ we have $P\left(\left|X_{k}\right|>\epsilon / d\right)=0$; i.e. for each $k \in \mathbf{N}, X_{k}=0$ with probability one. So, $\mu$ is degenerate which contradicts the assumption (iii). Thus $a_{n} \rightarrow 0$ after all.

b) Now let us show that $a_{n+1} / a_{n} \rightarrow 1$. Let $T_{n}:=a_{n} S_{n}+b_{n} \Rightarrow \mu$. Since, by the infinitesimality assumption (ii), $a_{n+1} X_{n+1} \rightarrow 0$ in probability, therefore (letting "lim" denote limit in distribution)

$$
\mu=\lim _{n \rightarrow \infty} T_{n+1}=\lim _{n \rightarrow \infty}\left(a_{n+1} S_{n}+b_{n+1}\right)=\lim _{n \rightarrow \infty}\left[\frac{a_{n+1}}{a_{n}} T_{n}+\left(b_{n+1}-b_{n} \frac{a_{n+1}}{a_{n}}\right)\right] .
$$

Hence the Convergence of Types Theorem (see e.g. Proposition 2.7.1 in Jurek and Mason (1993)) gives that $a_{n+1} / a_{n} \rightarrow 1$ and $b_{n+1}-b_{n} a_{n+1} / a_{n} \rightarrow 0$ as $n \rightarrow \infty$, which completes the argument for Step 1 .

Step 2. Now for what follows, let $c \in(0,1)$ be arbitrary but fixed.

For the sequence $\left(a_{n}\right)$ let us define

$$
m_{n}:=\max _{1 \leq k \leq n-1}\left\{k: \frac{a_{n}}{a_{k}} \leq c\right\} \text {, if such } k \text { exists, or } m_{n}:=1 \text { otherwise. }
$$

Then we have

$$
m_{n} \rightarrow \infty, \quad n-m_{n} \rightarrow \infty \text { and } \quad \frac{a_{n}}{a_{m_{n}}} \rightarrow c \quad \text { as } n \rightarrow \infty .
$$

Proof. This is so, because from the definition of $m_{n}$ we get

$$
\frac{a_{n}}{a_{m_{n}}} \leq c<\frac{a_{n}}{a_{m_{n}+1}} \quad \text { or } \quad 1 \leq c \frac{a_{m_{n}}}{a_{n}}<\frac{a_{m_{n}}}{a_{m_{n}+1}} \quad \text { for all sufficiently large } n \text {. }
$$


Step 3. For an infinitesimal triangular array $\left\{a_{n} X_{k}: 1 \leq k \leq n, n=\right.$ $1,2, \ldots\}$ (i.e. satisfying the condition (3)) there exists a non-increasing sequence of positive numbers $\delta_{1}, \delta_{2}, \ldots$, each $\leq 1$, such that

(i) $\delta_{n} \rightarrow 0$ as $n \rightarrow \infty$ and

(ii) $\forall(n \geq 1) \quad \forall(1 \leq k \leq n) \quad P\left(a_{n}\left\|X_{k}\right\| \geq \delta_{n}\right) \leq \delta_{n}$.

Proof. Simply note that (3) is equivalent to the following: $\left\|X_{n, j_{n}}\right\| \rightarrow 0$ in probability, for all $j_{n} \in \mathbf{N}$ such that $1 \leq j_{n} \leq n$.

Step 4. For the sequences $\left(m_{n}\right)$ from Step 2, and $\left(\delta_{n}\right)$ from Step 3, let us choose positive integers $q_{n}$ such that

$$
\begin{gathered}
q_{n} \leq \delta_{n}^{-1 / 2} \text { for all } n, \quad q_{n} \rightarrow \infty \text { as } n \rightarrow \infty, \text { and } \\
m_{n}+q_{n}<n \text { for all sufficiently large } n \geq 1
\end{gathered}
$$

Consequently, the partial sums $S_{n}$ can be written in three blocks

$$
S_{m_{n}}+\left(S_{m_{n}+q_{n}}-S_{m_{n}}\right)+\left(S_{n}-S_{m_{n}+q_{n}}\right)=S_{n} \text { for sufficiently large } n \geq 1 \text {. }
$$

Step 5. The rv's $V_{n}:=a_{n}\left(S_{m_{n}+q_{n}}-S_{m_{n}}\right)$ satisfy $\left\|V_{n}\right\| \rightarrow 0$ in probability as $n \rightarrow \infty$.

Proof. This is so, because for any $\epsilon>0$, applying Steps 3 and 4 , for all $n$ such that $\delta_{n}^{1 / 2}<\epsilon$ and $m_{n}+q_{n}<n$, we have that $\delta_{n} q_{n} \leq \delta_{n}^{1 / 2}<\epsilon$ and thus

$$
\begin{gathered}
P\left(\left\|V_{n}\right\| \geq \epsilon\right)=P\left(a_{n}\left\|\sum_{k=m_{n}+1}^{m_{n}+q_{n}} X_{k}\right\| \geq \epsilon\right) \leq \sum_{k=m_{n}+1}^{m_{n}+q_{n}} P\left(a_{n}\left\|X_{k}\right\| \geq \epsilon / q_{n}\right) \\
\leq \sum_{k=m_{n}+1}^{m_{n}+q_{n}} P\left(a_{n}\left\|X_{k}\right\| \geq \epsilon \delta_{n}^{1 / 2}\right) \leq \sum_{k=m_{n}+1}^{m_{n}+q_{n}} P\left(a_{n}\left\|X_{k}\right\| \geq \delta_{n}\right) \leq \delta_{n} q_{n} \leq \epsilon .
\end{gathered}
$$

Step 6. For the sequence $U_{n}:=\left(a_{n} / a_{m_{n}}\right)\left(a_{m_{n}} S_{m_{n}}+b_{m_{n}}\right)$ we have that

$$
U_{n} \Rightarrow T_{c} \mu \quad \text { and } \quad U_{n}+V_{n} \Rightarrow T_{c} \mu, \text { as } n \rightarrow \infty \text {. }
$$

Proof. First of all, by the assumption (iii) in the Theorem 1, and by Step 2 we have that $U_{n} \Rightarrow T_{c} \mu$. This with Step 5 (and e.g. Theorem 3.1 of Billingsley (1999)) proves the above claim.

Step \%. The family of probability distributions of the random variables

$$
W_{n}:=a_{n}\left(S_{n}-S_{m_{n}+q_{n}}\right)+b_{n}-\frac{a_{n}}{a_{m_{n}}} b_{m_{n}}, \quad n \geq 1,
$$


its tight. Equivalently, it is conditionally compact by the Prokhorov Theorem.

Proof. Note that using (7) we have that

$$
U_{n}+V_{n}+W_{n}=a S_{n}+b_{n} \Rightarrow \mu \text { as } n \rightarrow \infty .
$$

By Prokhorov Theorem (see e.g. Theorem 1.6.6 in Jurek and Mason (1993) or Theorem 2.10 in Araujo and Giné (1980)), using Step 6 and the formula (8), for a given $\epsilon>0$ there exist compact sets $K_{1}$ and $K_{2}$ such that

$$
P\left(-\left(U_{n}+V_{n}\right) \in K_{1}\right)>1-\epsilon / 2 \text { and } P\left(a_{n} S_{n}+b_{n} \in K_{2}\right)>1-\epsilon / 2
$$

for all $n \geq 1$. Since $W_{n}=\left(a_{n} S_{n}+b_{n}\right)+\left(-\left(U_{n}+V_{n}\right)\right)$ and

$$
\left\{a_{n} S_{n}+b_{n} \in K_{2} \text { and }-\left(U_{n}+V_{n}\right) \in K_{1}\right\} \subset\left\{W_{n} \in K_{1}+K_{2}\right\}
$$

we conclude that

$$
P\left(W_{n} \notin K_{1}+K_{2}\right) \leq P\left(-\left(U_{n}+V_{n}\right) \notin K_{1}\right)+P\left(a_{n} S_{n}+b_{n} \notin K_{2}\right) \leq \epsilon
$$

for all $n \geq 1$. Of course the set $K_{1}+K_{2}$ is compact by a standard argument. Thus the family of probability distributions of $\left(W_{n}\right)$ is tight (equivalently, conditionally compact in the weak convergence topology).

Step 8. The probability measure $\mu$ (in Theorem 1 ) satisfies the convolution equation $\mu=T_{c} \mu * \nu$ for some probability measure $\nu \in \mathcal{P}$.

Proof. In view of Step 7, there exists $\nu \in \mathcal{P}$ and a subsequence $Q \subset \mathbf{N}$ such that

$$
W_{n} \Rightarrow \nu, \quad \text { as } \quad n \rightarrow \infty \text { and } n \in Q
$$

From (8) and Steps 5 and 6 we conclude that

$$
U_{n}+W_{n} \Rightarrow \mu, \text { as } n \rightarrow \infty
$$

Since $q_{n} \rightarrow \infty$ therefore by the assumption (i) in Theorem 1 , we conclude

$$
\begin{gathered}
\alpha\left(\sigma\left(U_{n}\right), \sigma\left(W_{n}\right)\right) \leq \alpha\left(\sigma\left(X_{k}, 1 \leq k \leq m_{n}\right), \sigma\left(X_{k}, m_{n}+q_{n}+1 \leq k \leq n\right)\right. \\
\leq \alpha\left(\mathbf{X}, q_{n}+1\right) \rightarrow 0
\end{gathered}
$$

By (11), (9), Step 6, and Corollary 1 in the Appendix below

$$
U_{n}+W_{n} \Rightarrow T_{c} \mu * \nu \text { as } n \rightarrow \infty,
$$

which with (10) completes the proof of the Step 8. 
Finally, since our argument can be repeated for each $0<c<1$ (note that the distribution $\nu$ in Step 8 depends on $c$ ), we conclude by the convolution decomposition (5) that the measure $\mu$ is indeed selfdecomposable, which completes the proof of Theorem 1.

\section{Appendix.}

For ease of reference let us quote here

PROPOSITION 1. Let $X$ and $Z$ be two random elements on $(\Omega, \mathcal{F}, P)$ with values in a separable Banach space $E$ and let $U$ be a uniformly distributed on $(0,1)$ real valued random variable stochastically independent of $X$ and $Z$.

Suppose further that there exist $\epsilon>0, \delta>0$, a positive integer $N$, a Borel set $D \subset E$, and points $a_{1}, a_{2}, \ldots, a_{N}$ in $D$ such that

$$
P(X \in D)>1-\delta \quad \text { and } \forall(a \in D) \exists(1 \leq k \leq N)\left\|a-a_{k}\right\| \leq \epsilon .
$$

Then there exists an E-valued random variable $Y$ such that

(a) $Y$ is measurable with respect to $\sigma(X, Z, U)$,

(b) $Y$ is independent of $Z$,

(c) $Y \stackrel{d}{=} X$,

(d) $P(\|X-Y\|>2 \epsilon) \leq \delta+4 N^{1 / 2} \alpha(\sigma(X), \sigma(Z))$.

This is a corollary from Theorem 16.17 in Bradley (2007), volume 2, p. 139, for $S=E$ and $\mathcal{A}=\sigma(Z)$.

COROLLARY 1. Suppose $\left(X_{n}\right)_{n}$ and $\left(Z_{n}\right)_{n}$ be two sequences of E-valued random elements and $\mu, \nu$ be two probability Borel measures on $E$.

If $X_{n} \Rightarrow \mu$ and $Z_{n} \Rightarrow \nu$ as $n \rightarrow \infty$ and $\lim _{n \rightarrow \infty} \alpha\left(\sigma\left(X_{n}\right), \sigma\left(Z_{n}\right)\right)=0$ then

(a) for every $\epsilon>0$ there exists positive integer $n_{\epsilon}$ such that for every $n \geq n_{\epsilon}$ there exists an E-valued random variable $Y_{n}$ that is independent of $Z_{n}$ and satisfies both $Y_{n} \stackrel{d}{=} X_{n}$ and $P\left(\left\|X_{n}-Y_{n}\right\|>\epsilon\right)<\epsilon$.

(b) random elements $\left(X_{n}, Z_{n}\right) \Rightarrow \mu \times \nu$ and $X_{n}+Z_{n} \Rightarrow \mu * \nu$, as $n \rightarrow \infty$.

Proof. Applying the Prokhorov Theorem for the sequence $\left(X_{n}\right)_{n}$ we have that for a given $\epsilon>0$ there exists a compact set $K \subset E$ such that

$$
P\left(X_{n} \in K\right)>1-\epsilon / 2 \text { for all } n \in \mathbf{N} \text {. }
$$

From the compactness of $\mathrm{K}$, there are finitely points $a_{1}, a_{2}, \ldots, a_{M} \in K$ such that the open balls $B\left(a_{i}, \epsilon / 2\right), i=1,2, \ldots, M$ cover $\mathrm{K}$ and thus for any $x \in K$ there is an $a_{i}$ such that $\left\|x-a_{i}\right\|<\epsilon / 2$. 
Applying Proposition 1 for each pair $X_{n}, Z_{n}$ we get a random variable $Y_{n}$ such that $Y_{n}$ is independent of $Z_{n}, Y_{n} \stackrel{d}{=} X_{n}$ and

$$
P\left(\left\|X_{n}-Y_{n}\right\|>\epsilon\right) \leq \epsilon / 2+4 M^{1 / 2} \alpha\left(\sigma\left(X_{n}\right), \sigma\left(Z_{n}\right)\right) .
$$

Since $\lim _{n \rightarrow \infty} \alpha\left(\sigma\left(X_{n}\right), \sigma\left(Z_{n}\right)\right)=0$, we conclude that there exists a positive integer $n_{\epsilon}$ such that $P\left(\left\|X_{n}-Y_{n}\right\|>\epsilon\right)<\epsilon$ for all $n \geq n_{\epsilon}$. This completes the proof of part (a).

Part (b) follows from part (a) and Lemma 1.1 and Theorem 1.1 in Chapter 3 in Parthasarathy (1967).

\section{Literatura}

[1] A. Araujo and E. Gine (1980), The central limit theorem for real and Banach valued random variables, John Wiley \& Sons, New York.

[2] P. Billingsley (1999), Convergence of probability measures, Second Edition, John Wiley \& Sons, New York.

[3] R. C. Bradley (2007), Introduction to strong mixing conditions, vol. 1 and vol. 2, Kendrick Press

[4] Z. J. Jurek and J. D. Mason (1993), Operator-limit distribution in probability theory, John Wiley \& Sons

[5] Z. J. Jurek and W. Vervaat (1983), An integral representation for selfdecomposable Banach space valued random variables, Z. Wahrscheinlichkeitstheorie verw. Gebiete, vol. 62, pp. 247-262.

[6] K. R. Parthasarathy (1967), Probability measures on metric spaces, Academic Press, New York and London.

[7] M. Rosenblatt (1956), A central limit theorem and a strong mixing, Proc. Natl. Acad. Sci. USA vol. 42, pp. 43-47. 